\title{
Typography Design's New Trajectory Towards Visual Literacy for Digital Mediums
}

\author{
Stephen T.F. Poon \\ Correspondence: Stephen Poon, School of Media, Arts \& Design, Asia Pacific University of Technology \& Innovation, \\ Malaysia.
}

Received: November 5, 2020

Accepted: November 27, 2020

Online Published: December 14, 2020

doi:10.11114/smc.v9i1.5071

URL: https://doi.org/10.11114/smc.v9i1.5071

\begin{abstract}
Typographic elements have a huge impact on how designed mediums affect visual literacy. This paper reviews the principles, perspectives and approaches in the production and function of typographic design visual media (print and digital), with the aim to understand the relationship between typographic design on digital mediums, with the aim of examining its influence on people's ability to communicate ideas, meaning and messages effectively, while reflecting on its commercial implications for brands and marketers. Research via survey and a focus group implied there is a positive association between literacy and the application of graphic design and typography on digital communication mediums. Findings revealed that type design complements textual word elements to enhance cognition and understanding of messages. The integration of visual and texts facilitates reading, and for digital mediums, both legible layout and engaging typefaces are equally crucial. Graphic typeface for digital media, from smartphones to e-texts for learning, should apply visual hierarchy arrangement to achieve these objectives. Findings show typographic design is an essential aspect of social communication today, and digital designers play a fundamental role to enable audiences to improve their economic and social participation and gain its full advantages.
\end{abstract}

Keywords: typography design, visual literacy, type, typeface, digital media

\section{Introduction}

The era of web technologies has created unprecedented responses to digital media and visual communication. This is apparent in the design and innovation for an endless array of digital tools and resources. Technology, as an extension of post-industrial societies, has been observed and praised for its ability to construct new knowledge, new realities and social experiences and to develop various aspects of modern culture (Burnett, 2005).

It is evident that engaging visual design acts to ensure message efficiency, such as reading, consuming and exchanging information on screens and making connections with others using digital texts, images, emojis, multimedia content and diverse audio-visual artefacts (Reyna et al, 2008).

While some concerns are raised that the true potential behind digital and new media forms maybe buried under complex digital systems, others are more optimistic in their outlook. For example, the French sociologist Bruno Latour in 1996 (Burnett, 2005, p.125) predicted that man and machines would in future exchange relational positions: the virtual and real collide in and through participatory pursuits and activities.

In the $21^{\text {st }}$-century, evolving digital literacy skills are crucial for researchers considering how technology, media communication and design sectors boost participatory culture in society. With digital literacy and participation comes education: the knowledge disadvantaged are in hindered in their desire for socioeconomic improvements and transformation, e.g. access to government and public services, technological innovation, healthcare, education, civic involvement, social networking, and growth opportunities (Lankshear and Knobel, 2008).

Visual media and design communication involving visual intertextuality and literacy are thus important to clarify and examine to understand their transformative capabilities. However, some recent human-computer interaction studies show many web designers more inclined towards homogeneity in visual communication adaptation and commercial works, rather than demonstrating originality and skills in personalisation, due mainly to the two indicators of interface usability i.e. efficiency and effectiveness of web layouts (Alshaali, 2011).

Typically, contemporary digital media designers surmount challenges that are attributed to a poor grasp of aesthetic 
principles including weak or undistinguishable visual properties of clear type, colour, sizing, position and placement of elements such as brand logos. Confusing, chaotic or misaligned elements that do not adhere to hierarchy of connections lack strong conceptual design, distancing and frustrating audiences (Hashimoto and Clayton, 2009). Digital graphic typography that fatigues users' vision, accurate perception and concentration lead to a corresponding increase in design shortcuts, drop down menus and multi-tasking (Reyna et al, 2018).

\subsection{Objectives and Hypothesis}

To authenticate the contribution and role of visual design in improving digital literacy, this study aims to accomplish several objectives. The researcher's aim is to analyse the transformative capacities of contemporary digital media via the visual design process; specifically, the use of typography.

Several questions guide the researching process: First, how has technological evolution expanded the role of visual communication practices in contemporary design? Second, how does typography affect digital literacy? Third, how is graphic design valued for visual communication on digital media?

To provide a contextual framing of the study objectives, the hypothesis, there is a positive association between visual literacy and the application of uncluttered typography design on digital communication mediums, will be subject to critical evaluation and analysis. The next sections examine the body of literature on typography design's historical, commercial and educational roles in the evolutionary journey of print and digital visual communication mediums, in the context of improving visual literacy through typography.

\section{Literature Review}

Graphic design, according to Paul Rand (1993), is given to imply both "the process and product of [the] human imagination". The evolution of visual communication practices has had substantial influence on contemporary design through technological innovations. Multimedia learning has become the main means of message cognition and processing, in the social construction of complex new knowledge (Reyna et al, 2018). To understand the theoretical principles which elaborate on this subject, two significant publications will be overviewed: Graphic Communication Today by William Ryan and Theodore Conover and Typographic Design (Form and Communication) by Carter, Meggs and Day (2012).

Ryan and Conover (2004) examine the powerful sociological and psychological implications of typographic functions as visual communication medium and literacy resource for society. Carter, Meggs and Day (2012) assert the traditional, functionalist roles of typeface designs by tracing and establishing typography's influence in the service of visual communication. Calling typography an "interface", they believe that typographic legibility is an achievable outcome for designers equipped with critical problem-solving skills and a broad appreciation of the heritage, qualities and inherent attributes of typographic forms specimens in conveying surprising narratives and results for a range of graphic design challenges.

Establishing the rule of visual hierarchy as a "study of relationships of each part to the other and the whole" (Carter et al, 2012, p.90), enables design to combine equitable visual elements on the hierarchical structure to create a sense of spatial relationship based on relative dominance or subordinate characteristics of the elements. Other experimental aspects of typographic design research pioneered modern typographic innovations to demonstrate poetic power and potential through "legibility and transparency" of typography (Gere, 2008, pp.173-174).

This 'New Typography' swelled along the trajectory of digital media development such as video games, film special effects, virtual reality, multimedia, electronic music, graphics software and desktop publishing. Although initially perceived to be inversion of designers' self-expression and styles and labelled by critics as a disruptive culture marker, proponents saw digital graphics as tools which communicate a profound social celebration of technology as an amplifier of Western philosophies (Gere, 2008, pp.173-174)..

\subsection{Typography and the Screen}

Understanding typographic design in traditional print publishing is essential in appreciating typographic rules and functions (Miyatsu, 2018). Thomas J. Cobden-Sanderson, a $20^{\text {th }}$-century bookbinder and printer who crafted the Doves typeface with Emery Walker, zealously spoke up for the aesthetics of printing craftsmanship:

"The whole duty of Typography, as of Calligraphy, is to communicate to the imagination, without loss by the way, the thought or image intended to be communicated ..." (cited by Winckler, 1978, p.348).

Typographic design expanded its roles and functions through several developmental phases (Robertson, 2013) but it was during the early computing era where document pages were manipulated using WYSIWYG graphical interface systems (Alshaali, 2011; Shackel, 2009; Staples, 2000). As improvements were made in digital imaging technologies 
and standards, graphical interface systems were no longer limited to photomanipulation, but started extending to the compositing of images and type on single surfaces, such as the publishing of books and magazines using drawing tools such as Adobe Illustrator and Photoshop.

By the 1980s, MIT academics and Stanford University programmers had successfully reproduced digital alphabets by deconstructing Roman alphabets into a set of primitives. Digital typography developed among communities of engineers and computer interface scientists like Donald Knuth, embraced by type designers like Charles Bigelow and Kris Holmes (Lucida type creators). But it was CD-ROM's emergence in the late 1980s that seismically shifted visual culture, bringing display text upfront (Staples, 2000).

Modern digital visual culture principles were furthered by museum curator Ellen Lupton and J. Abbott Miller, American design educators and authors of Design, Writing, Research (1999), who premised their thesis on critical readings of the graphic designer's role in textual writing through intricately "arranging, sizing, framing and editing pictorial material, images and texts", breaking them down using grids, graphs, figures. In deconstructing the essence of language (making meaning), Lupton and Miller explored design's aesthetic capabilities to elaborate, direct and disrupt textual meaning via visual design and typographic layouts (in Poynor, 2003, p.124).

AIGA 2007 Gold Medal recipient Lupton believes that social constructionist approaches have been responsible for market democratisation in making typefaces freely available. This open source culture ensures typography remain "valuable, powerful and beautiful cultural tools", a gifting to humanity for commercial and educational purposes (Lupton, 2010). For existing typography to survive, conceptual designs tied to specific technology need to outlive the media per se. For typographic innovations to gain market traction, type specimens must therefore be technology-sensitive without being technology-subservient (Carter, 1993).

\subsection{Typography Design Functionality vs "Feelingvision"}

Wallace Kibbee (1948) pioneered the concept of modern typography as the simplification and legibility of forms that comply with the natural inclination of the human eye to seek harmony and ease. Reinterpreting this proclivity today, digital media designers create and manipulate in newer ways, crafting letterforms to revise "the very image of the printed word" either as traditional font on paper, or as pixels on screens (Staples, 2000).

Willi Kunz was a Swiss-born typographer based in New York (Graphic Design Archive, n.d.). He argued in Typography: Macro + Microaesthetics (2004) that older typesetting was the process by which the "collective relationships" between space and typographic marks become tangible and visible, every adjustment of space between words, lines and paragraphs symbolised designers' unique creativity in spatial and textual handling.

Among literacy issues, a discussion of "speed deficit" is relevant. This refers to habitual performance conditions when reading texts on websites and screens in comparison with reading on paper. Some longitudinal studies reviewed the rates of reading for digital and printed texts and framed the outcomes on efficiency aspects such as speed, accuracy, fatigue, comprehension and preference (Dillon, 1992; Fernandez et al, 2002). Though results were based on older technology systems, empirical evidence suggest that speed deficit for screen-based text reading are not attributed to factors such as line length, character spacing, text quantity, font size or style, perception manipulation or display criteria such as screen enhancements. Functional communication is based on why the message attracts and how useful and comprehensible the information is.

Readability and appearance of traditional print depends on designers' acute perceptivity about spatial emotions, to differentiate the "awkwardly squeezed, tight, scattered, enlivening" and etc. aesthetics of typefaces from ineffective layouts, requiring strong eye judgement as "spacing type relies on optical sensitivity" (Kunz, 2004, cited in Cullen, 2012, p.86).

Rhetorical attributes of meanings, characters, attitudes, atmosphere, connotative, semantically quality and type personalities have further been mapped by design researchers using theoretical frameworks which relate typefaces to a range of human emotions (Brumberger, 2003; Koch, 2011). Eva Brumberger (2003) gathered empirical data on typography's rhetorical attributes of professionalism, violence and friendliness, and found that typeface design intersections of emotional (cultural, connotative) and rational (verbal characteristics) helps facilitate readers' understanding of visual language.

Similarly, Koch (2011) collected empirical and qualitative evidence demonstrating that despite their contribution to visual communication, overlapping variables in typefaces imply that values, meaning attributions and behavioural factors such as feelings arousal are individualised and not typically generalisable. Colour is another dimension of affective design which shifts meaning, temperature and value of verbal texts (Carter, Day and Meggs, 2012, p.83; Samara, 2007, p.255). The emotive components of colour are associated to one's experience at an instinctual and biological level, and affected by cultural views (Ryan and Conover, 2004, p.168). 
In Paul Miller and Svitlana Matviyenko (2014)'s recent anthology on app computing, The Imaginary App, social theorist Brian Massumi's concept of feelingvision is cited as the illusory experience of enjoining a tactile form with a visual event. This new form of perceptual experience could bring creative levels of human expression in "forceful, affective manner ... [which] recompose the relations between our senses" (p.120). Notwithstanding literature demonstrating contextual difficulties in recognising, analysing and evaluating emotions from visual stimuli, the attitudinal, social and behavioural aspects of visual communication in design research is crucial to understand how visual data act as "scaffolds" for human thought and emotional expressions and behaviours.

\subsection{Effects of Print and Digital Media on Design Communication}

Visuals are processed 60,000 times faster than text (Vogel, Dickson and Lehman, 1986). Intuitive visual content facilitates reading, information recall, understanding and memory. For branding and advertising sectors, improved retention from visual recall leads to positive perception and loyalty for brands and products (Ryan and Conover, 2004, p.17).

Devices such mobile phones, tablets and smartphones spur new literacies today. Rich environments such as multimedia, digital video, user generated content (blogs, vlogs) and applications (apps) prove increasing digital knowledge sophistication, offering opportunities for advertisers and brand marketers to communicate directly with consumers.

Compared to traditional media like television, screens engender personalisation and multi-channel participation. Interactive mediums leverage on visual literacy to engage attention increase channel interest and affect behavioural changes. Digital billboards, for instance, have expanded graphic design capabilities in social communication, including the transmittal of brand messages (Leiss et al, 2005, p.19). Nevertheless, the ethics of digital persuasion presents imminent challenges today, as some web design experiences predicate unwary clicking among users seeking instant gratification (Reile, 2016). Consumer psychology studies on print medium's sensory impact track its ability to communicate in evoking or heightening emotions, stimulating retention and recall, and improving rates of brand recognition, legitimising marketing and branding tactics. Media specialists today, as they did in the era of print catalogues and mail-order shopping, continue to rally for print's advantages in establishing brand presence, captivating niche markets or target audiences (Dooley, 2010; Leiss et al, 2005). Thus, the main responsibility of typographic designers lies in a fundamental grasp of tangible cognitive experiences.

Digital audiences' intellectual, emotional and higher order thinking skills have reached a point where unifying informational hierarchy and understanding of visual symbols and concepts interplay on screens. Graphic designing on web-based platforms offers online ideas, inspirations and a range of tools enabling sketching, mock-ups, and in-fill dimensions of colours, layout and spaces to be projected and presented.

Digital designers seek simple, legible typefaces to produce composite images and texts on a single surface; this simplification of forms changes the nature of type and layout preferences. Handcrafted, cursive forms have gone the way of pre-industrial printing presses. As interface developer Harshita Arora (2018) explains, except for the use of Grotesque typefaces to suit specific thematic messages, Serif and San-Serif are the two popular modern typeface families that exhibit legibility and readability best from less complex effects. Digital media platforms have liberated graphic artists from the hand-lettering and carving skills once highly valued. Advancing technologies democratise once-mechanical printing, word processing, layout design and typesetting, while software packages and application tools enable a wider range of creative multimedia visual representations and possibilities in print and interactive mediums, as Neely (2012) notes:

“... there are almost as many methods of graphic design as there are graphic designers! Seeing a designer's personality shine through in their work is always a bonus".

\subsection{Whither Visual Literacy for Digital Readability}

Recent digital and new media scholarship started to critically address the different challenges in the use of digital engagement methods such as visual, video, audio and text as sensorial stimulation for audiences in developing accurate comprehension and cognition for information processing (especially reading); these same issues have taken the education, publications and communications sectors by storm (Lankshear and Knobel, 2008).

Digital literacy must be evaluated in the context of evolving technologies. Studies showing the positive effects of learners' adaptation to interactive media, with visual-based apps and games are increasingly used by educators to help learners improve reading proficiency through personalised environments (Ash, 2013; Reyna et al, 2018). Other cutting-edge visual media such as Augmented Reality (AR) have the capacity to enhance digital competencies (Lankshear and Knobel, 2008; Media Smarts, n.d.).

Dr Ron Burnett (2005) of the Emily Carr University of Art and Design states, "images are no longer just representations or interpreters of human actions, [but are] central to every action that connects humans to each other and to technology 
[...] as much reference points for information and knowledge as visualisations of human creativity" (p.xiv). Goodfellow (2011) claims that research on visual media literacies has been limited to evaluating user competencies based around popular culture identifications among niche communities, and less on how critical skills such as reading complement digital literacy. Lead and Jones (cited in Goodfellow, 2011, p.7) suggest that students in the digital era show higher inclination to participate in textual genres that apply hybridised technologies and complex applications such as mediated contexts, for e.g. learning through video. This current state of situated literacies could produce literacy crisis in future, with the gradual decline in learners' abilities to produce, enact, synthesise and evaluate academic skills and aptitudes. Many academics are sceptical of incorporating, adapting and realigning digital skills for teaching purposes, since the cultural attributes associated with growing digital literacy among students infer a need of multiple literacies, including visual, in navigating the "new communications order" (Goodfellow, 2011, p.2).

Print's tangibility, engagement and credibility are strengths which digital media lack, but evangelists of the latter argue that digital's forte lies in storytelling, visual-primed narratives, low-cost brand exposure and wider audience reach with sensorial appeals of visual, audio and video content (Miller and Matviyenko, 2014). Despite this, reading facilitation for screens such as page-turning and scrolling tools offer little added benefit unless it triggers efficient and effective comprehension. Invariably, getting verbal to coexist with visual poses a problem, particularly for web and interactive mobile or apps designers (Alshaali, 2011, p.11), visual stimulation enables emotional response and gratification for screen users by creating focus on digital environments.

Readability of typeface is a fundamental criterion when designing layouts like textbooks and e-learning texts, and visual hierarchy ensure maximum legibility and emotional response for print audiences (Samara, 2007). Brumberger and Northcut (2013, pp.89-90), for instance, argue that the gold standard behind the application of "sans serif fonts for headings, serif fonts for body text" interplays with visual emotions: headers need the formality of sans serif to gain reader's immediate attention, but longer blocks of main texts is best served by subtler serif fonts.

For online and screen-based typefaces, an inversion of this golden rule is suggested, where san serif best serves the purpose of textual consistency (Arora, 2018). Despite the disparities, legibility awareness of spatiality, dots, lines, squares, textures and layout means designers must make intuitive considerations in combining typefaces and images with text to facilitate reading. In this regard, Samara (2007, p.226) notes:

"The results of poorly integrated type and images fall into two categories. First is type that has nothing in common with images and is completely separated from image areas. Second, typography that aggressively integrates with image that it becomes an illegible mass of shape and texture".

\section{Research Methodology}

To examine the impact of design in facilitating literacy of content on digital media, and how this has expanded visual communication practice, a mixed-methods research with quantitative and qualitative evaluation was chosen, comprising a focus group and survey questionnaire sampling of participants from targeted audience segment.

Taking Ryan and Conover (2004)'s assumption that effective integration of verbal (textual) and visual (graphic) elements in media design facilitates literacy, a perception study was carried out using survey and focus group to gather data from among the growing segment of student users of smartphones and digital media devices in urban Malaysia. Mixed-methods research enables quantification of data and results to gain insights on respondents' attitudes, perceptions and preferences. The survey questionnaire was presented in multiple choice, attitudinal scale and closed-ended format. The instrument was designed to understand the attitudes and perceptions of the audience affected by digital literacy, aged between 17 to 25 years, mainly Malaysian high school and tertiary students. In total, 115 survey participants were sampled, while four students were chosen to participate in a focus group session. Results of the survey and focus group were tabulated and are presented for analysis and discussion in the following section.

\section{Findings of the Study}

Media literacy requires visually appealing and functional types that do not lose legibility when operating on digital screens. This study was designed to gather a range of views and opinions of survey respondents, with seven questions prepared in the online questionnaire, some with accompanying visuals.

The first question of owning devices was aimed at validating claims that digital technology is essential to modern lifestyles, $64 \%$ of the respondents own a smartphone, $1 \%$ own a tablet or iPad only; $32 \%$ own both smartphone and tablet, while 3\% are not device owners. For the next question, the choices of text and picture, respondents were asked to choose which layout they prefer (Figure 1 and Figure 2). They were then asked which aspect of layout and design attracted them, $93 \%$ of respondents answered those visuals were the prime attraction, while the remaining $7 \%$ preferred text. A very similar result was found when asked which was easier to read, it showed all sampled respondents answered that Figure 1 was easier to read than Figure 2. 


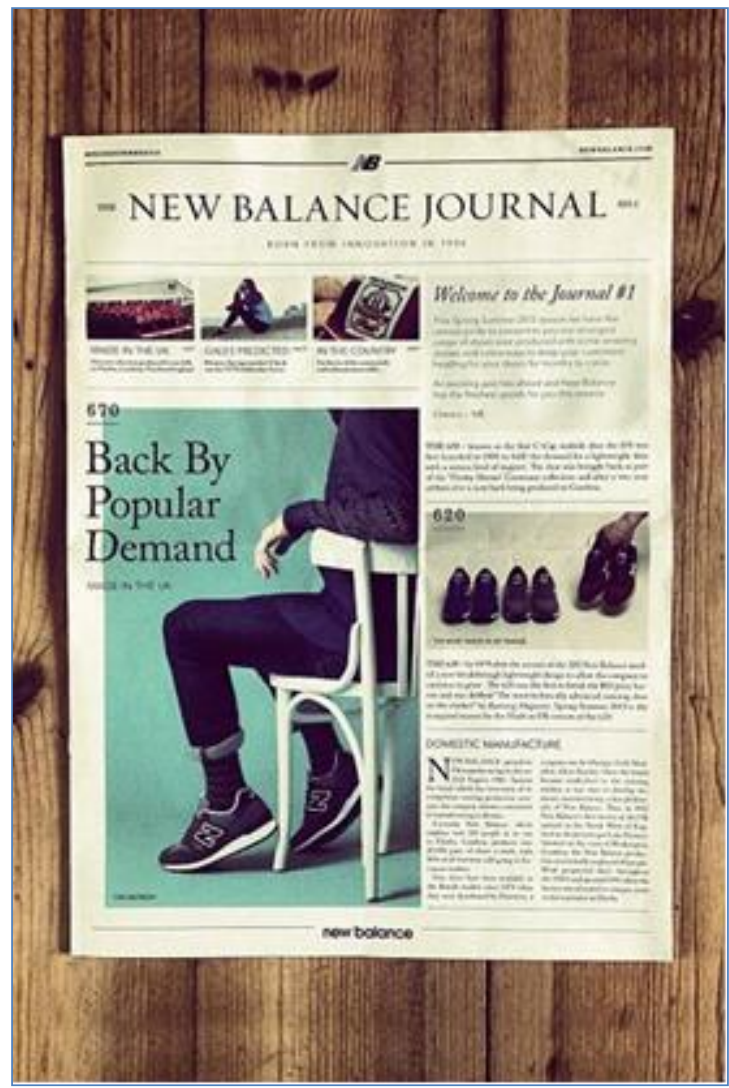

Figure 1. Cover Page, the New Balance Journal 1

This suggests that visualisation has become the new aesthetic in what Massumi terms the feelingvision dimension (cited in Lupton and Miller, 1999). Images as visual stimulation form have immediacy and allure, improving desire to engage. The focus group respondents stated they were primarily attracted by the coloured type element, while white spaces surrounding the text catch attention.

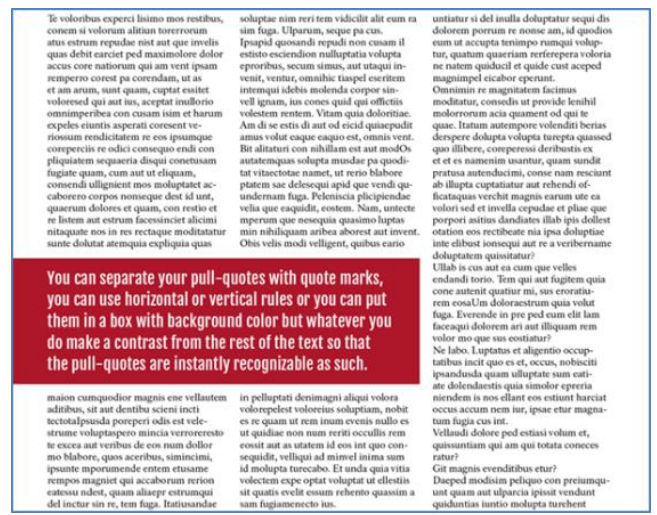

Figure 2. Pull-quotes, Magazine Designing

Question sought to understand respondents' perception towards the importance of layout design for the purpose of reading. Results are presented whereby the majority said layout is very important for reading; but 8 respondents did not think so. Respondents generally believe good layout facilitates content processing as keywords are clearly shown.

Question of utilised an attitudinal scale, designed to find out how much respondents agreed that digital technology had contributed to reading ease. It shows $19 \%$ of respondents strongly agreeing that digital technology has greatly contributed to reading; $65 \%$ expressed agreement, $13 \%$ were neutral, and 3\% disagreed. This finding showed the habitual preference among majority of respondents to read through digital devices like smartphones, laptops and tablets to gather information and messages. 


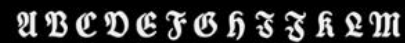
กOPQRGT $\mathfrak{X} \mathfrak{W} \mathfrak{X} \mathfrak{Z}$ a b c de f g b i j k I m

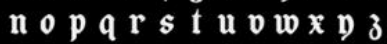
$\$ £ € 0123456789$

Figure 3. Coop Blackletter

Question subsequent asked respondents to choose between two type fonts, Blackletter (Figure 3) and Calibri (Figure 4) for the purpose of reading, Blackletter's cursive hand-lettered form is an antiquated typeface from the European Renaissance era, originating from the Latin script system, while Calibri font has subtly rounded stems and corners; the modern type is clearly observable at larger sizes and distances, even for casual reading.

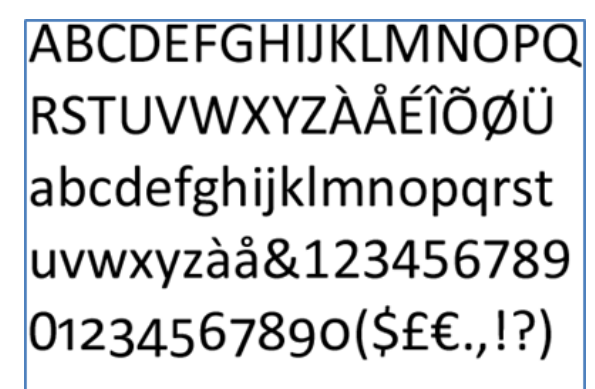

Figure 4. Calibri, Font Packager

From the question of comfortable for reading, all survey respondents voted for Calibri (Figure 4) as the type font they prefer for the purpose of reading. This shows an overwhelming preference for typefaces which are clear, legible and comfortable to read.

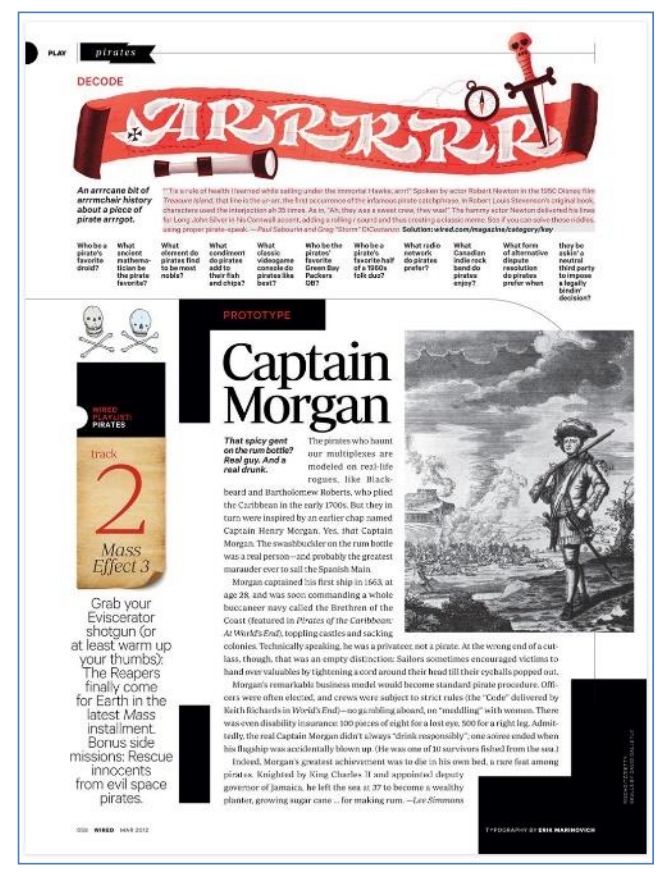

Figure 5. Pirate Magazine Page in Wired

Question of picture is more effective in giving out messages was aimed at finding out respondents' preference between two choices of layout design given, each with visual (images) and text (words) incorporated (Figure 5 and Figure 6), 81\% of respondents think Figure 6 is more effective in sending the message, and 19\% of respondents answer that Figure 5 is more effective, which implies detailed descriptive information in verbal text alone may not reach its optimal effectiveness. 


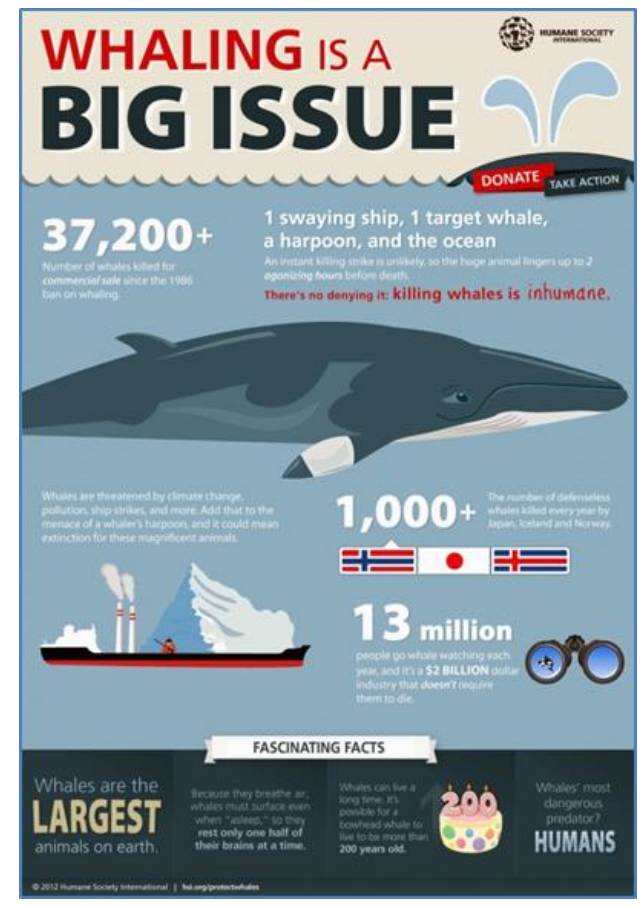

Figure 6: Whaling Is A BIG Issue

Graphical interface fulfils its functionality for audiences to whom visuals act as a 'warm-up', an invitation to read and understand textual message. However, this finding also implies that layout design can be an attraction factor in respondents' perceptions. The next section synthesises the findings and discusses various implications and limitations of research.

\section{Data Analysis and Discussion}

As digital literacy skills involve producing, applying and evaluating visual appeals to convey information and meaning, effective typography enhances textual cognition, which in turn, expands technology's potential in advancing visual practice. The inherent power of visual communication on digital media supplants the presence of text alone in facilitating reading among digital natives.

Results from the survey show that an integration of verbal and visual is the best way to communicate. In this study, perceptions towards design are an important consideration for audiences; suggesting that typography in content design facilitates understanding via ease of reading. Text placement without spatial comfort hinders message comprehensibility and engagement. However, layout becomes peripheral for those who value reading written text, to whom the central task of reading surmounts every other visual consideration.

In traditional media, the linear processes involved in reading texts is limited to an ability to produce meaning, sentence after sentence. For digital media, typography and layout work complementarily to make reading effective: clarity, comfort and visual appeal are inducements for users not predisposed to reading textual information. Designing type and digital layouts must factor in user-friendliness, ease of use and adaptability to audiences. Type sizing, spacing and font alignments are becoming near-effortless undertakings with software which can create document templates, typefaces and clip arts, either purchased online or freely downloaded. Microsoft Word outgrew being typing software to incorporate a suite of layout functions, including art, colours, shapes, page styles and background settings that visually express messages through annotations and diagrams.

In this study, respondents viewed Blackletter form negatively, noting its weak readability compared to Calibri, and perceive its use mainly for decorative purposes. Visuals affect cognitive accuracy as value judgements are unavoidable in guiding perceptions. To resolve this, annotations, copy lines or descriptive captions in clear type should accompany images.

With digital screens occupying significant places in social lifestyles and interactions, the "swipe-to-right" factor has given rise to finger-fast devices with visual appeal, proof that people need attention-grabbing elements. The survey validates the hypothesis that users prefer uncluttered, appealing typefaces as attributes of efficiency and effectiveness of visual designs. With increasing reliance on feelingvision to interpret human moods and needs, the usage of visual icons (emoticons) in lieu of text may nevertheless be a source of growing concern among academics, families and child development experts, as emoticon and emoji dependency may lower individuals' intelligence in social communication 
by reducing our innate abilities to say something effectively founded on logical reasoning and real emotions.

From the survey conducted, overall findings indicated that emergent digital technologies have added another narrative function to typography, namely, to design legible texts that enable the social construction of new knowledge, and, just as on print, interactive mediums need a balanced typographic environment to communicate its objectives. Digital media require visually appealing and functional types that do not lose legibility on screens.

The study of Malaysian students' adaptation to digital mediums and how typeface facilitates readability of messages seem to point towards this notion. Suffused daily with phenomenal quantities of visual communication, research insight has specific implications for the Malaysian digital consumer markets, reflecting national statistics on the growing importance of economic and social participation in e-commerce and e-government (MCMC, 2018). From a design educator's perspective, the importance of typographic legibility must be perceived as a complementary value addition to multimedia (tablets, laptops, mobile devices and smartphone apps) and type design must augment users' engagement and interest.

Some shortcomings were noted in the process of conducting research.

- The comparisons were not effectively weighted, as objects were unequal in value with their counterpoint. For instance, Figure 1 shows the headline placed prominently on the front page and Figure 2 is a body text display. It emphasises the fact that textual designs affect responsiveness, although serving different purposes. The function of large images and headlines is to create immediacy and urgency for attention, while body text provides textual information for the reading audience.

- Typefaces were not equally weighted. Print media serif type in Figure 3 and digital media san serif type in Figure 4 are of unequal value, although this essentially emphasises the differences between perceptions of typeface functionality for print and digital media.

- Layout designs (Figures 5 and 6) were unequal in counterpoint value. It is suggested that future research measure levels of readability and audience engagement based on visual layout design comparisons of equal counterpoint value, either for print or digital media.

\section{Conclusions}

In this paper, the impact of type design on reading and literacy was studied. Findings from survey and focus group suggest the integration of visual and text in designing layouts ensures stronger message transmittal effectiveness and outcomes. Designers of apps, multimedia, gaming platforms, educational/learning products and websites need to take digital literacy development no less seriously than commercial interests by optimising typographic innovations for information transmittal, while acknowledging negative behavioural aspects and preferences of consumers.

With its seamless interactivity, digital media has grown to be the obvious No.1 communication medium of an urban generation. Typographic design enables efficient transmission of information and social messages, which presents designers with great opportunities and unique challenges ahead. Predictably, the eventual displacement and obsolescence of traditional graphic designing practices would occur (Koch, 2011; Lupton, 2004). Images and type play complementary roles in digital literacy, image to exemplify, type to describe. Functional type is a necessary interface between textuality and literacy, and while the debate is far from settled, typography need greater industrial acknowledgement in its role as digital media's "partner-in-crime".

The integration of visual and verbal seem the most effective way to strengthen perceptual outcomes in cognitive processing, message retention and affecting sensorial experiences (Koch, 2011). Essentially, digital media specialists must anticipate and promote typography design's future importance in augmenting human connections and expressions, by showing that typographic development lead the way to digital literacy and richer social communication. Today's digital media designers must be ready to wear multiple hats to craft meaningful and engaging experiences for different audiences, and in doing so, dignifies typography practice as a fundamental service to society.

\section{Acknowledgements}

Writing this paper has truly been an experience that I will not soon forget. I am grateful for the selfless guidance that I received from my cluster. Particularly, I offer my sincerest gratitude to Pinky Khoo for her linguistics support and advice, also for suggesting alternative modes of thought when I found myself at a creative impasse. I am thankful to my family and friends have given this work so much support that it would be invidious to list names; with one exception.

\section{References}

Alshaali, S. K. R. (2011). Human-Computer Interaction: Lessons from Theory and Practice. [Doctoral Thesis]. University of Southampton School of Management. 
Arora, H. (2018, January 16). How Typography Determines Readability: Serif vs. Sans Serif, and How to Combine Fonts. Free Code Camp. Retrieved August 05, 2020 from https://www.freecodecamp.org/news/how-typography-determines-readability-serif-vs-sans-serif-and-how-to-combi ne-fonts-629a51ad8cce/

Ash, K. (2013). Tablets, Smartphones Influence Reading Instruction. Retrieved June 24, 2020 from www.edweek.org/dd/articles/2013/02/06/02reading.h06.html

Brumberger, E. R. (2003). The Rhetoric of Typography: The Persona of Typeface and Text. Technical Communication, 50(2) May: 206-223. Retrieved August 27, 2020 from http://rid.olfo.org/doc/rhetoftypography.pdf

Brumberger, E. R., \& Nothcut, K. (2013). Designing Texts: Teaching Visual Communication. Amityville, New York: Baywood Publishing Co Inc. https://doi.org/10.2190/DTT

Burnett, R. (2005), How Images Think. Cambridge, Massachusetts: MIT Press. https://doi.org/10.7551/mitpress/3580.001.0001

Carter, M. (1993). Reputations: Matthew Carter. Eye Magazine, 11(3). Retrieved June 16, 2020 from www.eyemagazine.com/feature/article/reputations-matthew-carter

Carter, R., Day, B., \& Meggs, P. (2012). Typographic Design: Form and Communication. $5^{\text {th }}$ edition. Hoboken, New Jersey: John Wiley \& Sons, Inc.

Cullen, K. (2012). Design Elements: Typography Fundamentals. Beverly, Massachusetts: Rockport Publishers.

Dillon, A. (1992). Reading from Paper versus Screens: A Critical Review of the Empirical Literature. Ergonomics, 35(10), 1297-1326. https://doi.org/10.1080/00140139208967394

Dooley, R. (2010, October 04). Paper Beats Digital for Emotion. Neuroscience. Retrieved March 29, 2020 from http://www.neurosciencemarketing.com/blog/articles/paper-vs-digital.htm

Fernandez, E., Alfaro, A., Tormos, J. M., Climent, R., Martınez, M., Vilanova, H., \& Pascual-Leone, A. (2002). Mapping of the human visual cortex using image-guided transcranial magnetic stimulation. Brain Research Protocols, (10), 115-124. https://doi.org/10.1016/S1385-299X(02)00189-7

Gere, C. (2008). Digital Culture. $2^{\text {nd }}$ edition. Islington, London: Reaktion Books.

Goodfellow, R. (2011). Literacy, Literacies and the Digital in Higher Education. Teaching in Higher Education, 16(1), 131-144. https://doi.org/10.1080/13562517.2011.544125

Graphic Design Archive (n.d.). Willi Kunz. Rochester Institute of Technology [online], RIT Libraries. Retrieved August 27, 2020 from https://library.rit.edu/gda/designers/willi-kunz

Hashimoto, A., \& Clayton, M. (2009). Visual Design Fundamentals: A Digital Approach. Rockland, Massachusetts: Charles River Media.

Kibbee, W. (1948). Modern Trends in Typography. American Journal of Economics and Sociology, 7(2), 215-220. https://doi.org/10.1111/j.1536-7150.1948.tb00677.x

Koch, B. E. (2011). Human Emotion Response to Typographic Design. [Dissertation]. University of Minnesota.

Kunz, W. (2004). Typography: Macro- And Microaesthetic. $2^{\text {nd }}$ edition. Teufen, Switzerland: Arthur Niggli.

Lankshear, C., \& Knobel, M. (2008). Digital Literacies: Concepts, Policies and Practices. New York: Peter Lang Publishing, Inc.

Leiss, W., Kline, S., Jhally, S., \& Botterill, J. (2005). Social Communication in Advertising: Consumption in the Mediated Marketplace. ( $3^{\text {rd }}$ ed.). Abingdon, London: Routledge.

Lupton, E. (2010). Free Font Manifesto. Retrieved March 22, 2020 from http://elupton.com/2010/01/free-font-manifesto/

Lupton, E. (2004). Thinking with Type. New York: Princeton Architectural Press.

Lupton, E., \& Miller, J. A. (1999) Design, Writing, Research. New York: Phaidon Press.

Malaysia Communications and Multimedia Commission, MCMC (2018) e-Commerce Consumers Survey 2018 [Report]. Retrieved August 04, 2020 from https://www.mcmc.gov.my/skmmgovmy/media/General/pdf/ECS-2018.pdf

MediaSmarts. (n.d.). Digital Literacy Fundamentals. Canada's Centre for Digital and Media Literacy. Retrieved April 25, 2020 from http://mediasmarts.ca/digital-media-literacy-fundamentals/digital-literacy-fundamentals 
Miller, P. D., \& Matviyenko, S. (2014). The Imaginary App. Cambridge, Massachusetts: The MIT Press. https://doi.org/10.7551/mitpress/9820.001.0001

Miyatsu, R. (2018, July 23). Cobden-Sanderson and the Doves Press. Washington University in St Louis. Retrieved August 13, 2020 from https://library.wustl.edu/cobden-sanderson-and-the-doves-press/

Neely, A. (2012). How Technology has Influenced Graphic Design. Retrieved March 02, 2020 from www.brighthub.com/multimedia/publishing/articles/121403.aspx

Poynor, R. (2003). No More Rules: Graphic Design and Postmodernism. London: Laurence King Ltd.

Rand, P. (1993). Design, Form, and Chaos. New Haven, Connecticut: Yale University Press.

Reile, W. (2016, September 27). User Gratification: The Digital Dopamine for E-Commerce. Prisync. Retrieved August 29, 2020 from https://blog.prisync.com/digital-dopamine/

Reyna, J., Hanham, J., \& Meier, P. (2018). The Internet explosion, digital media principles and implications to communicate effectively in the digital, space. E-Learning \& Digital Media, 15(1), 36-52. https://doi.org/10.1177/2042753018754361

Robertson, F. (2013). Print Culture: From Steam Press to e-Book. London: Routledge. https://doi.org/10.4324/9780203144206

Ryan, W. E., \& Conover, T. E. (2004). Graphic Communications Today. $4^{\text {th }}$ edition. Florence, Kentucky: Delmar Learning/Thomson Learning.

Samara, T. (2007). Design Elements: A Graphic Style Manual. Beverly, Massachusetts: Rockport Publishers.

Shackel, B. (2009). Human-computer interaction: Whence and wither? Interacting with Computers, 21(5/6), 353-366. https://doi.org/10.1016/j.intcom.2009.04.004

Staples, L. (2000). Typography and the Screen: A Technical Chronology of Digital Typography, 1984-1997. Design Issues, 16(3), Autumn; pp.19-34. https://doi.org/10.1162/07479360052053306

Vogel, D. R., Dickson, G. W., \& Lehman, J. A. (1986). Persuasion and the Role of Visual Presentation Support: The UM/3M Study. Working Paper (June). University of Minnesota, Management Information Systems Research Center.

Winckler, P. A. (1978). Reader in the History of Books and Printing. London: Information Handling Services (IHS Markit Ltd).

\section{Images Credit}

Figure 1: Cover Page. (2012) The New Balance Journal 1 [image]. Retrieved March 06, 2020 from http://hypebeast.com/2012/7/the-new-balance-journal-issue-1

Figure 2: Pull-quotes (2013). Magazine Designing [image]. Retrieved April 17, 2020 from $\mathrm{http} / / / \mathrm{www}$.magazinedesigning.com/pull-quotes/

Figure 3: Jacque, A. (2014). Coop Blackletter [image]. Retrieved from May 28, 2020 from https://cdn.myfonts.net/s/aw/original/388/0/198739.png

Figure 4: Calibri. (2013). Font Packager [image]. Retrieved June 09, 2020 from http://forum.xda-developers.com/google-nexus-5/themes-apps/app-fontster-betas-flashable-font-packs-t2513985

Figure 5: Marinovich, E. (n.d.). Pirate Magazine Page in Wired. Pinterest [image]. Retrieved March 10, 2020 from https://www.pinterest.com/pin/504825439453434087/

Figure 6: Humane Society International. (2012). Whaling Is A BIG Issue. Retrieved July 21, 2020 from http://www.hsi.org/issues/whaling/facts/infographic.html

\section{Copyrights}

Copyright for this article is retained by the author(s), with first publication rights granted to the journal.

This is an open-access article distributed under the terms and conditions of the Creative Commons Attribution license which permits unrestricted use, distribution, and reproduction in any medium, provided the original work is properly cited. 